\title{
Nuclear import of Nkx2-2 is mediated by multiple pathways
}

Wenbo Lin ${ }^{1 \#}$, PengPeng Xu ${ }^{1 \#}$,YingYing Guo ${ }^{1}$, Qingjie $\mathrm{Jia}^{1}$, and Tao Tao ${ }^{1 *}$

${ }^{1}$ Xiamen University School of Life Sciences, Xiamen University, Xiamen, Fujian 361005, China;

${ }^{*}$ Corresponding author:

Dr. Tao Tao

E-mail: taotao@xmu.edu.cn

\# These authors contributed equally to this work. 


\section{Abstract}

Nkx2-2 homeoprotein is essential for the development of the central nervous system and pancreas. Although the nuclear localization signals of Nkx2-2 have been identified, the responsible transport receptor is still unknown. Here, we demonstrate that imp a1 not only interacts with Nkx2-2 but also transports it into the nucleus in vitro by acting together with imp $\beta 1$. However, the nuclear import of Nkx2-2 in cells was not inhibited in response to knockdown expression of endogenous imp $\beta 1$ or over-expression of Bimax2. Furthermore, imp $\beta 1$ and imp 13, but not imp 4, directly interact with Nkx2-2 and are capable of transporting Nkx2-2 in an in vitro import assay. By GST pull-down assay, we demonstrate that mutation of NLS1 or NLS2 has no effect on interaction with imp $\alpha 1$ or imp 13 , but significantly reduced binding to imp $\beta 1$. Thus, the nuclear import of Nkx2-2 is mediated not only by the classical import pathway but also directly by imp $\beta 1$ or imp 13.

Keywords: Nkx2-2, importin $\alpha$, importin $\beta$, nuclear localization signal, homeodomain

\section{Introduction}

Nkx2-2 plays an important role in the development of the CNS and pancreas, [1,2]. Nkx2-2 functions as a transcription repressor by recruitment of co-repressor Groucho 3 (Grg3) [3] or Grg4 [4], but it also contains a transcriptional activation domain (TAD), and this domain is repressed by the conserved "Nk2-specfic domain" (NK2-SD) [5]. In addition, Nkx2-2 contains a homeodomain, which is not only responsible for its DNA binding activity [5], but also governs the subcellular distribution of this protein [6]. Each extremity of the homeodomain of Nkx2-2 contains a nuclear localization signal (NLS). NLS1 is composed of ${ }^{127} \mathrm{KKRKRR}^{132}$, and NLS2 is composed of ${ }^{180}{ }^{R Y K M K R A R}{ }^{187}$; these sequences cooperatively mediate the nuclear localization of Nkx2-2 [6].

The nuclear transport of the proteins is signal-dependent and occurs through the nuclear pore complex (NPC) via importin $\beta$ proteins $[7,8]$. Nuclear localization signals are divided into two classes: classical and non-classical NLSs. Classical NLSs are initially recognized by imp $\alpha$, and the latter interact with imp $\beta 1$ to forming a ternary complex, the bound cargo proteins 
subsequently traverse the NPC then are released in the nucleus after association with RanGTP, which is concentrated in the nucleus [9]. Several subtypes of imp as have been identified in mammals, and these proteins can be separated into three subfamilies on the basis of sequence conservation [10]. Bimax2, a designed peptide, specifically inhibits the imp $\alpha / \beta 1$-dependent nuclear import pathway due to its much higher affinity to imp as [11]. In addition to the classical nuclear import pathway, a cargo with a non-classical NLS is recognized directly by imp $\beta s$, and then transported into nucleus, e.g. imp $\beta 1$ is an import receptor for smad3 and SRY, and imp 13 is responsible for the nuclear transport of pax6, Arx and myopodin [8].

Although NLSs have been identified within Nkx2-2, the responsible shutting carrier remains unknown. In the present study, we determined that imp $\alpha 1$, but not imp $\alpha 3$ or imp $\alpha 7$, directly interacts with and transports Nkx2-2 into the nucleus. However, co-expression of Bimax2 with Nkx2-2 did not repress the nuclear transport of Nkx2-2, as well as knockdown expression of imp $\beta 1$ in cells, suggested that the nuclear import of Nkx2-2 was not solely mediated by classical import pathway in cells. Thus, imp $\beta 1$ and imp 13 were examined and confirmed to interact with Nkx2-2 and to transport this protein into the nucleus. Interaction between $\mathrm{Nkx2-2}$ and imp $\alpha 1$, imp 13 or imp $\beta 1$ was differently dependent on NLS1/2 within Nkx2-2.

\section{Materials and methods}

\section{Cell culture}

HeLa, NIH3T3 and 293T cells were grown in Dulbecco's modified Eagle's medium (\#SH30022.01B, HyClone, USA) supplemented with 10\% fetal bovine serum and incubated at $37^{\circ} \mathrm{C}$ in a $5 \% \mathrm{CO}_{2}$, air incubator.

\section{Construction of expression plasmids}

Polymerase chain reaction (PCR) to amplify murine Nkx2-2 was conducted with LA Taq (\#DRR20AG, Takara, Japan). The resultant fragments were inserted into pEGFP-C1 or pET28a. The plasmid expressing Bimax2 or $\beta$-galactosidase (gal)-EGFP was kindly provided by Dr. Yanagawa or Dr. Seger [11,12]. NLS $S_{S 440} \beta-$-gal-EGFP and Nkx2-2- $\beta$-gal-EGFP were constructed by coupling with the corresponding coding region at the Nhel and Hindlll sites on the basis of $\beta$-gal-EGFP. To generate the DsRed-GST-NLS $S_{S 40}$ and 
dsRed-GST-Nkx2-2 plasmids, pDsRed1-C1 was inserted in tandem with the coding region for GST and Nkx2-2 or NLS SV40. Control shRNA or shRNA for imp $\beta 1$ was constructed by inserting the corresponding sequences at the Hpal and Xhol sites of pLL3.7. Oligonucleotide sequences are listed in Supplementary Table S1.

\section{Protein expression and purification}

All proteins were expressed in the BL21(DE3) E. coli strain. Expression of GST-tagged Nkx2-2-EGFP, imp as and imp $\beta$ s were induced by $1 \mathrm{mM}$ isopropyl- $\beta$-D-thiogalactoside (IPTG) for $4 \mathrm{~h}$ at $25^{\circ} \mathrm{C}$. His-tagged Nkx2-2 and its mutants, Ran, RanQ69L, NTF2, RanBP1, and RanGAP1 were induced for $4 \mathrm{~h}$ at $25^{\circ} \mathrm{C}$ with $0.5 \mathrm{mM}$ IPTG. The cells were collected and lysed by sonication in lysis buffer (50 mM HEPES(pH 7.5), 100 mM NaCl, 0.5\% Nonidet P-40, 10\% glycerol, $1 \mathrm{mM}$ dithiothreitol, $1 \mathrm{mM}$ phenylmethylsulfonyl fluoride(PMSF), soluble GST-Nkx2-2-EGFP and His-tagged proteins were isolated using glutathione Sepharose 4B beads (GST beads, \#6200226, Bio-Rad, USA) or Ni-NTA beads(\#1560131, Bio-Rad, USA) according to the manufacturer's instructions. Purified proteins were desalted with a PD10 desalting column (\#17-0851-01, GE healthcare, USA) at $4^{\circ} \mathrm{C}$. The concentration of the purified proteins was measured using TCA kits (\#23225, Thermo Fisher Scientific, USA) according to the manufacturer's instructions.

\section{GST pull-down assay}

GST or GST-tagged imp $\alpha 1$, imp $\alpha 3$, or imp $\alpha 7(5 \mu \mathrm{g})$, or imp $\beta 1$, imp 4, or imp $13(1 \mu \mathrm{g})$ were immobilized on GST beads at $4^{\circ} \mathrm{C}$ overnight, then washed 3 times with lysis buffer and incubated with $0.2 \mu \mathrm{g}$ of $\mathrm{His}_{6}-\mathrm{Nkx2}-2$ and/or mutants at $4^{\circ} \mathrm{C}$ for $1.5 \mathrm{~h}$. The beads were subsequently centrifuged and washed twice. The binding complexes were separated by using SDS-PAGE. Nkx2-2 and its mutants were detected with an anti-His antibody (1:2000, SC803, Santa Cruz, USA), or the complexes were divided into two equal portions, which were incubated with $5 \mu \mathrm{M}$ RanGDP or RanQ69LGTP for another $3 \mathrm{~h}$ at $4^{\circ} \mathrm{C}$, then the complexes were loaded onto SDS-PAGE gels for western blotting analysis to detect for dissociation of impßs and Nkx2-2. 
To perform GST pull-down assays in cells, GST or GST-Nkx2-2 was overexpressed in 293T cells, and the cell lysate was incubated with GST beads for $3 \mathrm{~h}$ at $4^{\circ} \mathrm{C}$. The beads were subsequently collected and washed twice. The bound proteins were separated by using SDS-PAGE and detected with anti-imp $\alpha 1$ (1:2000, No:10819-1-AP, Proteintech, China), anti-imp $\beta 1$ (1:5000, No:10077-1-AP, Proteintech, China), anti-imp 4 (1:2000, No:11679-1-AP, Proteintech, China) and anti-imp 13 [13] antibodies.

\section{Co-immunoprecipitation}

HA-imp a1 was co-expressed in 293T cells with EGFP-Nkx2-2 or EGFP-Nkx2-2 $\triangle \mathrm{HOX}$. Subsequently, the cells were lysed, and the supernatants were precleared with protein G-plus agarose (SC500778, Santa Cruz, USA) for $2 \mathrm{~h}$ at $4^{\circ} \mathrm{C}$. Then supernatants were incubated with $1 \mu \mathrm{g}$ of an anti-HA antibody (No:51064-2-AP, Proteintech, China) and $10 \mu \mathrm{l}$ of protein G-plus agarose at $4^{\circ} \mathrm{C}$ for $4 \mathrm{~h}$. The interaction complex was then separated by using SDS-PAGE and detected with anti-HA (1:8000, No:51064-2-AP, Proteintech, China) and anti-GFP (1:2000, SC9996, Santa Cruz, USA) antibodies.

\section{In vitro import assay}

Briefly, HeLa cells were grown on $8^{\star} 8 \mathrm{~mm}$ glass coverslips, then washed twice with transport buffer (20 mM HEPES-KOH ( $\mathrm{pH} 7.3$ ), $110 \mathrm{mM}$ potassium acetate, $5 \mathrm{mM}$ magnesium acetate, $1 \mathrm{mM}$ EGTA, $2 \mathrm{mM}$ dithiothreitol, and 250 $\mathrm{mM}$ sucrose). Permeabilization was done by using $40 \mu \mathrm{g} / \mathrm{ml}$ digitonin (\#D5628, Sigma, USA) for $5 \mathrm{~min}$ on ice. The permeabilized cells were incubated with 25 $\mu \mathrm{l}$ of the transport reaction mixture for $30 \mathrm{~min}$ at either $30^{\circ} \mathrm{C}$ or $4^{\circ} \mathrm{C}$. Each $25 \mu \mathrm{l}$ of the transport reaction mixture contained $100 \mathrm{nM}$ GST-Nkx2-2-EGFP cargo protein and $1 \mu \mathrm{M}$ imp $\beta 1$, either alone or together with $1 \mu \mathrm{M}$ imp $\alpha 1$, imp $\alpha 3$ or imp $\alpha 7$, or $1 \mu \mathrm{M}$ imp 4 or imp 13, and supplemented with an energy regenerating system [14]. The import reaction was terminated by the addition of $500 \mu \mathrm{l}$ of TB buffer. The cell nuclei were stained with DAPI. GFP-fusion proteins were detected by Nikon Eclipse 80i fluorescence microscope. 


\section{Immunofluorescence staining}

NIH3T3 cells were grown on glass coverslips and transfected with indicatied expressing plasmids for $24 \mathrm{~h}$. Cells were then fixed with $3.7 \%$ formaldehyde, and permeabilized with PBS supplemented with $0.1 \%$ Triton X-100. The cells were incubated with primary antibodies overnight at $4{ }^{\circ} \mathrm{C}$, and subsequently incubated with secondary antibodies for $1 \mathrm{~h}$ at room temperature. Cells were then mounted and visualized with a Nikon Eclipse 80i fluorescence microscope.

\section{shRNA treatment}

HeLa cells were first grown on $60 \mathrm{~mm}$ plate, and treated with control shRNA or imp $\beta 1$ shRNA for $48 \mathrm{~h}$. To detect endogenous imp $\beta 1$ by western blotting, the cells were harvested and analyzed by the anti-imp $\beta 1$ antibody, $\beta$-actin was used as loading control and detected by anti- $\beta$-actin (1:5000, \#A5316, Sigma, USA) antibody. To detect endogenous imp $\beta 1$ via immunofluorescence staining, cells were trypsinized and seed on glass coverslips. Then, cells were collected and stained for imp $\beta 1$ as mentioned above. To detect the subcellular

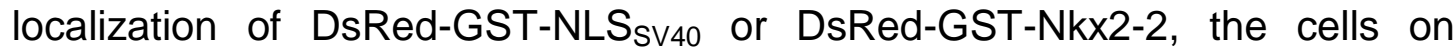
coverslips were transfected with indicated plasmids for another $24 \mathrm{~h}$. The cells were fixed and visualized with a Nikon Eclipse 80i fluorescence microscope. Nuclear staining was conducted by using DAPI.

\section{RESULTS}

\section{Importin $\alpha 1 / \beta 1$ interacts with and transports Nkx2-2 into the nucleus}

NLSs within Nkx2-2 have been reported [6]; however, the responsible import receptor is still unknown. The identified two NLSs are almost identical to classical class I and II monopartite NLSs [6,15] (Fig. 1A). Thus, NLS1 or NLS2 was fused with $\beta$-gal-EGFP to construct NLS1- $\beta$-gal-EGFP or NLS2- $\beta$-gal-EGFP, and the subcellular localization of these fusion proteins

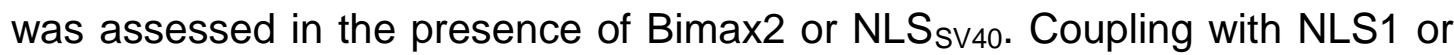
NLS2 induced nuclear localization of these fusion proteins, even in the

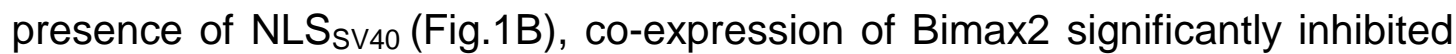


the nuclear import of both fusion proteins. This result indicated that the nuclear import of NLS1 or NLS2 occurs via a classical nuclear import pathway. Because Nkx2-2 contains only these two NLSs [6], the nuclear transport of Nkx2-2 also appeared to be mediated by an imp $\alpha / \beta 1$ heterodimer. Imp as are divided into three subfamilies, and a representative member of each subfamily (imp $\alpha 1$, imp $\alpha 3$, and imp $\alpha 7$ ) was therefore used in these experiments. To determine which imp a protein might be involved in the nuclear import of Nkx2-2, GST pull-down assays were conducted. GST-tagged impa1, impa3 or impa7 was immobilized on GST beads and incubated with purified $\mathrm{His}_{6}-\mathrm{Nk} 22-2$. Recombinant His $_{6}-\mathrm{Nkx2}-2$ interacted with imp $\alpha 1$, but not with imp a3 or imp a7 or GST alone (Fig 2A). To confirm this interaction in cells, Co-IP assay was conducted. HA-imp a1 was co-transfected into 293T cells with EGFP-Nkx2-2 or the negative control EGFP-Nkx2-2 $\triangle \mathrm{HOX}$, which lacks both NLSs. EGFP-Nkx2-2, but not EGFP-Nkx2-2 $\Delta$ HOX, coprecipitated with HA-imp a1 (Fig. 2B). Because Nkx2-2 contains two NLSs, we sought to determine whether one or both NLSs were required in association with imp a1. To this end, one or both NLSs were inactivated by site-directed mutagenesis, and the interaction between imp $\alpha 1$ and Nkx2-2 or Nkx2-2 mutants was detected. Imp a1 was able to interact with NLS1 or NLS2 mutant of Nkx2-2 but not with double mutation of NLS1 and NLS2 (Fig 2C). These results indicated that the interaction of Nkx2-2 with imp a1 is dependent on its NLSs. To confirm the involvement of the classical imp $\alpha / \beta 1$ import pathway in the nuclear transport of Nkx2-2, an in vitro nuclear import assay was conducted. Nkx2-2 was present in the cytoplasm in the absence of importin proteins when cells were incubated at $30^{\circ} \mathrm{C}$, and the addition of imp $\alpha 1 / \beta 1$ resulted in transport of Nkx2-2 into the nucleus. However, imp $\alpha 3 / \beta 1$ or imp $\alpha 7 / \beta 1$ did not transport Nkx2-2 into the nucleus. The nuclear import of Nkx2-2 was inhibited at $4^{\circ} \mathrm{C}$, even in the presence of imp $\alpha 1 / \beta 1$, imp $\alpha 3 / \beta 1$ and imp $\alpha 7 / \beta 1$ (Fig. 2D). These results suggested that the nuclear import of $N k \times 2-2$ is mediated by a classical nuclear import pathway in vitro.

\section{Nuclear transport of Nkx2-2 is not solely dependent on classical import pathway in cells}


To verify whether the imp $\alpha 1 / \beta 1$ heterodimer also functions in the nuclear import of Nkx2-2 in cells, Bimax2, a potent inhibitor of classical nuclear import pathway[11], was co-expressed with Nkx2-2- $\beta$-gal-EGFP in NIH3T3 cells, and the subcellular localization of $\mathrm{Nkx2-2}$ was subsequently observed, $\mathrm{NLS}_{\mathrm{SV} 40}-\beta$-gal-EGFP was used as a positive control. NLS $S_{S V 40}-\beta$-gal-EGFP was primarily localized in the nucleus in the absence of Bimax2, but blocked in cytoplasm by Bimax2, surprisingly, the nuclear localization of Nkx2-2 was not affected when Bimax2 was co-expressed (Fig. 3A), indicating that nuclear import of Nkx2-2 in cells was not solely dependent on the classical import pathway. To further confirm this possibility, the expression of imp $\beta 1$ in HeLa cells was knocked down by transfection with an shRNA targeting imp $\beta 1$, which also disrupts the imp $\alpha / \beta 1$-mediated nuclear import pathway [16]. Endogenous imp $\beta 1$ was clearly decreased after treatment with imp $\beta 1$ shRNA, as confirmed through either immunofluorescence or immunoblotting assays (Fig. 3B), and the nuclear localization of dsRed-GST-NLS ${ }_{S v 40}$, a well-known imp $\alpha / \beta 1$-recognized cargo, was impaired in imp $\beta 1$ knockdown cells, but the nuclear localization of dsRed-GST-Nkx2-2 was not affected (Fig. 3C). These results suggested that the nuclear import of $\mathrm{Nkx2-2}$ in cells is not solely mediated by the classical imp $\alpha / \beta$ pathway.

\section{Importin $\beta 1$ and importin 13 directly interact with Nkx2-2}

Because Nkx2-2 was not transported solely through the classical nuclear import pathway, we sought to determine whether (and which) imp $\beta$ proteins are involved in the nuclear import of Nkx2-2. Because imp $\beta 1$ and imp 13 mediate the nuclear transport of homeoproteins [17], the interaction of these proteins with Nkx2-2, together with imp 4, was examined. To this end, GST-tagged imp $\beta 1$, imp 4 or imp 13 was immobilized on GST beads and incubated with $\mathrm{His}_{6}-\mathrm{Nkx2}-2$. Nkx2-2 interacted with imp $\beta 1$ and imp 13, but not imp 4 or GST alone (Fig. 4A). To verify whether the interaction between imp $\beta$ s and Nkx2-2 was sensitive to RanGTP, complexes of imp $\beta 1$, imp 4 or imp 13 with Nkx2-2 were further incubated with RanGDP or RanQ69LGTP. The interaction between imp $\beta 1$ or imp 13 and Nkx2-2 was clearly decreased in the presence of RanQ69LGTP, in contrast to treatment with RanGDP, whereas GST or GST-imp 4 displayed no interaction with Nkx2-2 (Fig. 4B). To confirm 
the interaction of imp $\beta 1$ or imp 13 with Nkx2-2 in cells, GST or GST-Nkx2-2 was over-expressed in 293T cells and subsequently immobilized on GST beads, the interacting partner was detected with the respective antibodies. Endogenous imp $\beta 1$ and imp 13 interacted with GST-Nkx2-2, but not with GST, whereas endogenous imp 4 did not interact with either GST or GST-Nkx2-2 (Fig. 4C). These results indicated that imp $\beta 1$ or imp 13 interacted with Nkx2-2. To confirm that the interaction of imp $\beta 1$ or imp 13 with Nkx2-2 was dependent on the NLSs, a GST pull-down assay was conducted to detect the interaction between imp $\beta 1$ or imp 13 and Nkx2-2 or its NLS mutants. Nkx2-2 interacted with imp $\beta 1$ or imp 13, mutation of either NLS1 or NLS2 reduced interaction with imp $\beta 1$, but not with imp 13; however, both mutation of NLS1/2 abolished their interaction (Fig. 4D), suggesting that imp $\beta 1$ or imp 13 binding to Nkx2-2 is differently dependent on NLSs.

\section{Importin $\beta 1$ and importin 13 transport Nkx2-2 into the nucleus}

Although imp $\beta 1$ and imp 13 bound to Nkx2-2 both in vitro and within cells, whether they transport Nkx2-2 into the nucleus remained obscure. To address this question, recombinant imp $\beta 1$, imp 4 and imp 13 were tested in an in vitro nuclear import assay. GST-Nkx2-2-EGFP was detected in the cytoplasm in the absence of importin $\beta$ proteins. In contrast, GST-Nkx2-2-EGFP was detected in the nucleus in the presence of either imp $\beta 1$ or imp 13, but not in the presence of imp 4, and no GST-Nkx2-2-EGFP was detected in the nucleus when cells were incubated at $4^{\circ} \mathrm{C}$ (Fig. 4E). Therefore, imp $\beta 1$ and imp 13 transport Nkx2-2 into the nucleus in vitro.

\section{Discussion}

Nkx2-2 homeoprotein is important for the development of the pancreas and the CNS [1,2]. After synthesis in the cytoplasm, this protein is transported into the nucleus, where it exerts its function. In the present study, we found that the nuclear import of Nkx2-2 was mediated not only by the classical imp $\alpha 1 / \beta 1$ heterodimer but also directly by imp $\beta 1$ or imp 13. By using either GST pull-down or Co-IP assays, we demonstrated that imp $\alpha 1$, imp $\beta 1$ or imp 13 directly binds to Nkx2-2, and their interactions are differently dependent on NLS1 and NLS2. Moreover, they transport Nkx2-2 into the nucleus in in vitro import assays (Fig. 2, 4). Hence, we demonstrated that both classical and 
non-classical nuclear import pathways are involved in the nuclear import of Nkx2-2. The usage of multiple import pathways for individual cargo has been reported before, e.g. core histones and Rev are transported into nucleus via importin $\beta 1$, transportin, importin 7 , and importin $9[18,19]$. In the present study, we found that knockdown expression of imp $\beta 1$, or inhibition classical import pathway by Bimax2, did not affect nuclear import of Nkx2-2 in cells (Fig. 3), indicating that the import pathways for Nkx2-2 are redundant in cells, as seen in the example of Dicer [20].

In a previous study, we have summarized the nuclear import pathway of homeoproteins and found that these proteins are transported into the nucleus either by imp $\alpha / \beta 1$ heterodimer, e.g. Brn2, Oct3/4, or by imp $\beta$ s, e.g. Arx, pax6 [17]. The first step of the classical nuclear import pathway is imp a's recognition of the classical NLS, a peptide rich in basic amino acids that is present within most imp $\alpha / \beta 1$ cargo homeoproteins, such as Yox1p $\left({ }^{34} K_{K R R R R}{ }^{39}\right)$, Brn2 $\left({ }^{355} \mathrm{RKRKKR}^{360}\right)$, Oct3/4 $\left({ }^{223} \mathrm{RKRKR}^{237}\right)$ and Oct6 $\left({ }^{336} \mathrm{RKRKKR}{ }^{341}\right)$, mutation of these peptides impair their nuclear localization [21]. These peptides are similar to NLS1 $\left({ }^{127} \mathrm{KKRKRR}^{132}\right)$ of Nkx2-2. In present study, NLS2 of Nkx2-2, was also found to induce nuclear transport of $\beta$-gal-EGFP via the classical nuclear import pathway (Fig. 1). Both NLS1 and NLS2 interact with imp $\alpha 1$, mutation of either one did not reduce their interaction (Fig. 2), suggesting that they may bind to imp $\alpha$ independently, a similar result was observed in the case of imp 13 (Fig. 4D), however, intact Nkx2-2 was required for interaction with imp $\beta 1$, as mutation of either NLS significantly reduced their interactions (Fig. 4), these results reconfirm that imp $\alpha 1$ or imp $\beta 1$ directly interact with $\mathrm{Nkx2-2}$ individually, and nuclear transport of Nkx2-2 could be mediated by different import pathways. Comparison with the other homeodomain-containing cargo of imp $\beta 1$ and imp 13 revealed characteristic binding specificity of different homeoproteins, e.g. deletion of either basic region of pax6 reduce binding to imp13 [22], but has no effect on Nkx2-2 interaction; and a peptide similar to NLS2 of Nkx2-2 within PDX1 may be required for binding to imp $\beta 1[23,24]$, but both NLSs are required for $\mathrm{Nkx2-2}$ interaction with imp $\beta 1$. It is interesting to find that nuclear import of Nkx2-2 was also mediated by imp $\beta 1$ and imp 13 in the context of 
Nkx2-2, when NLS1/2 function alone as classical NLS, thus suggesting that the residues spanning NLSs also contribute to interaction with imp $\beta 1$ or imp 13. NLS1 and NLS2 locate at each terminus of homeodomain, which fold into a helix-turn-helix structure, we suspect that this structure facilitates interaction with imp $\beta s$.

In conclusion, we demonstrated that the nuclear import of Nkx2-2 is mediated by classical and non-classical import pathways.

\section{Acknowledgments:}

This work was supported by grants from the Ministry of Science and Technology of China (\#2012CB966602 and \#2013CB910803 to Dr. Tao Tao) and the National Science Foundation of Fujian (\#2013J05054 to Dr. Wenbo Lin).

\section{References}

[1] J. Briscoe, L. Sussel, P. Serup, D. Hartigan-O'Connor, T.M. Jessell, J.L. Rubenstein, J. Ericson, Homeobox gene Nkx2.2 and specification of neuronal identity by graded Sonic hedgehog signalling, Nature 398 (1999) 622-627.

[2] L. Sussel, J. Kalamaras, D.J. Hartigan-O'Connor, J.J. Meneses, R.A. Pedersen, J.L. Rubenstein, M.S. German, Mice lacking the homeodomain transcription factor Nkx2.2 have diabetes due to arrested differentiation of pancreatic beta cells, Development 125 (1998) 2213-2221.

[3] M.J. Doyle, Z.L. Loomis, L. Sussel, Nkx2.2-repressor activity is sufficient to specify alpha-cells and a small number of beta-cells in the pancreatic islet, Development 134 (2007) 515-523.

[4] J. Muhr, E. Andersson, M. Persson, T.M. Jessell, J. Ericson, Groucho-mediated transcriptional repression establishes progenitor cell pattern and neuronal fate in the ventral neural tube, Cell 104 (2001) 861-873.

[5] H. Watada, R.G. Mirmira, J. Kalamaras, M.S. German, Intramolecular control of transcriptional activity by the NK2-specific domain in NK-2 homeodomain proteins, Proc Natl Acad Sci U S A 97 (2000) 9443-9448.

[6] B. Hessabi, I. Schmidt, R. Walther, The homeodomain of Nkx2.2 carries two cooperatively acting nuclear localization signals, Biochem Biophys Res Commun 270 (2000) 695-700.

[7] A.M. Tartakoff, T. Tao, Comparative and evolutionary aspects of macromolecular translocation across membranes, Int J Biochem Cell Biol 42 (2010) 214-229.

[8] Y.M. Chook, K.E. Suel, Nuclear import by karyopherin-betas: recognition and inhibition, Biochim Biophys Acta 1813 (2011) 1593-1606.

[9] I.G. Macara, Transport into and out of the nucleus, Microbiol Mol Biol Rev 65 (2001) 
570-594, table of contents.

[10] D.S. Goldfarb, A.H. Corbett, D.A. Mason, M.T. Harreman, S.A. Adam, Importin alpha: a multipurpose nuclear-transport receptor, Trends Cell Biol 14 (2004) 505-514.

[11] S. Kosugi, M. Hasebe, T. Entani, S. Takayama, M. Tomita, H. Yanagawa, Design of peptide inhibitors for the importin alpha/beta nuclear import pathway by activity-based profiling, Chem Biol 15 (2008) 940-949.

[12] D. Chuderland, A. Konson, R. Seger, Identification and characterization of a general nuclear translocation signal in signaling proteins, Mol Cell 31 (2008) 850-861.

[13] T. Tao, J. Lan, G.L. Lukacs, R.J. Hache, F. Kaplan, Importin 13 regulates nuclear import of the glucocorticoid receptor in airway epithelial cells, Am J Respir Cell Mol Biol 35 (2006) 668-680.

[14] J.M. Mingot, S. Kostka, R. Kraft, E. Hartmann, D. Gorlich, Importin 13: a novel mediator of nuclear import and export, EMBO J 20 (2001) 3685-3694.

[15] S. Kosugi, M. Hasebe, N. Matsumura, H. Takashima, E. Miyamoto-Sato, M. Tomita, H. Yanagawa, Six classes of nuclear localization signals specific to different binding grooves of importin alpha, J Biol Chem 284 (2009) 478-485.

[16] R. Pawlowski, E.K. Rajakyla, M.K. Vartiainen, R. Treisman, An actin-regulated importin alpha/beta-dependent extended bipartite NLS directs nuclear import of MRTF-A, EMBO J 29 (2010) 3448-3458.

[17] W. Ye, W. Lin, A.M. Tartakoff, T. Tao, Karyopherins in nuclear transport of homeodomain proteins during development, Biochim Biophys Acta 1813 (2011) 1654-1662.

[18] P. Muhlhausser, E.C. Muller, A. Otto, U. Kutay, Multiple pathways contribute to nuclear import of core histones, EMBO Rep 2 (2001) 690-696.

[19] M. Arnold, A. Nath, J. Hauber, R.H. Kehlenbach, Multiple importins function as nuclear transport receptors for the Rev protein of human immunodeficiency virus type 1 , J Biol Chem 281 (2006) 20883-20890.

[20] M. Doyle, L. Badertscher, L. Jaskiewicz, S. Guttinger, S. Jurado, T. Hugenschmidt, U. Kutay, W. Filipowicz, The double-stranded RNA binding domain of human Dicer functions as a nuclear localization signal, RNA 19 (2013) 1238-1252.

[21] N. Yasuhara, N. Shibazaki, S. Tanaka, M. Nagai, Y. Kamikawa, S. Oe, M. Asally, Y. Kamachi, H. Kondoh, Y. Yoneda, Triggering neural differentiation of ES cells by subtype switching of importin-alpha, Nat Cell Biol 9 (2007) 72-79.

[22] J.E. Ploski, M.K. Shamsher, A. Radu, Paired-type homeodomain transcription factors are imported into the nucleus by karyopherin 13, Mol Cell Biol 24 (2004) 4824-4834.

[23] G. Guillemain, G. Da Silva Xavier, I. Rafiq, A. Leturque, G.A. Rutter, Importin beta1 mediates the glucose-stimulated nuclear import of pancreatic and duodenal homeobox-1 in pancreatic islet beta-cells (MIN6), Biochem J 378 (2004) 219-227.

[24] B. Hessabi, P. Ziegler, I. Schmidt, C. Hessabi, R. Walther, The nuclear localization signal (NLS) of PDX-1 is part of the homeodomain and represents a novel type of NLS, Eur J Biochem 263 (1999) 170-177. 


\section{Figure legends}

\section{Fig. 1 The NLSs of Nkx2-2 function as classical NLSs}

A. NLS1 or NLS2 of murine Nkx2-2 was aligned with class I or class II NLSs, where $X$ indicates any residue.

B. Immunofluorescence or fluorescence images of transfected NIH3T3 cells expressing $\beta$-gal-EGFP alone (a) or NLS1- $\beta$-gal-EGFP (c, f) with Flag-tagged NLS $\mathrm{SV}_{40}$ or Bimax2 (d, g) or NLS2- $\beta$-gal-EGFP (i, I) with Flag-tagged NLS $S_{S v 0}$ or Bimax2 $(j, m)$.

Fig. 2 Importin a1 interact with Nkx2-2 and transport it into the nucleus by acting with imp $\beta 1$

A. C GST or GST-imp as were immobilized on GST beads, and subsequently tested for interaction with recombinant $\mathrm{His}_{6}-\mathrm{Nkx2}-2$ (A) or together with Nkx2-2 mutants $(C)$. The input and bound fractions were analyzed by SDS-PAGE and subsequent by CBB staining or immunoblotting with an anti-His antibody.

B. The supernatants of $293 \mathrm{~T}$ cells co-expression of HA-imp $\alpha 1$ and GFP-Nkx2-2 or GFP-Nkx2-2 $\Delta$ HOX were tested for co-immunoprecipitation with an anti-HA antibody. The input and precipitated complex was separated by using SDS-PAGE and subsequent immunoblotting with anti-HA or anti-GFP antibodies.

D. An in vitro import assay showing that the nuclear import of Nkx2-2 is dependent on imp $\alpha 1 / \beta 1$, but not imp $\alpha 3 / \beta 1$ and imp $\alpha 7 / \beta 1$, when incubated at $30^{\circ} \mathrm{C}(\mathrm{b}, \mathrm{c}, \mathrm{d})$. GST-Nkx2-2-EGFP was not detected in the nuclei when incubated at $4^{\circ} \mathrm{C}(e, f, g)$.

Fig. 3 Nuclear import of Nkx2-2 in cells is not solely mediated by the classical nuclear import pathway

A. Immunofluorescence or fluorescence images of NIH3T3 cells expressing $N_{S V 40} \beta-$-gal-EGFP $(a, c)$ or Nkx2-2- $\beta$-gal-EGFP $(f, h)$ together with 
Flag-Bimax2 (d, i). The nuclei were stained with DAPI (g, j).

B. HeLa cells were treated with control shRNA or imp $\beta 1$ shRNA for $72 \mathrm{~h}$. The cells were collected and tested for imp $\beta 1$ expression by using immunofluorescence (a, d, left panel) or immunoblotting (right panel) with an anti-imp $\beta 1$ antibody. In the left panel, the transfected cells are shown in green $(b, e)$, and the nuclei were stained with DAPI (c, f). In the right panel, $\beta$-actin was used as a sample loading control.

C. HeLa cells were treated with the control shRNA or imp $\beta 1$ shRNA for $48 \mathrm{~h}$, then transfected with plasmids to express DsRed-GST-NLS or DsRed-GST-Nkx2-2 for another $18 \mathrm{~h}$, and subsequently fixed for visualization. A green signal indicates transfected cells $(b, e, h, k)$, and Nkx2-2 or NLS $S_{S 40}$ are shown in red $(a, d, g, j)$. The nuclei were stained with DAPI $(c, f, i, l)$

Fig. 4 imp $\beta 1$ and imp 13, but not imp 4, directly interact with Nkx2-2 and transport it into nucleus

A. D GST or GST-tagged imp $\beta$ s were immobilized on GST beads and detected for interaction with $\mathrm{His}_{6}-\mathrm{Nkx2}-2(\mathrm{~A})$ or Nkx2-2 mutants (D). The input and bound fractions were analyzed by using SDS-PAGE and subsequently CBB staining or immunoblotting with an anti-His antibody.

B. The complexes of imp $\beta$ s and Nkx2-2 were divided into two portions and incubated with RanGDP or RanQ69LGTP to detect for dissociation of the complex. The input and bound fractions were analyzed by using SDS-PAGE and subsequent CBB staining or immunoblotting with an anti-His antibody.

C. GST or GST-Nkx2-2 was expressed in 293T cells and immobilized on GST beads, the interaction partners were separated by using SDS-PAGE and detected with anti-imp $\beta 1$, anti-imp 4 or anti-imp 13 antibodies.

E. An in vitro import assay showing that the nuclear import of Nkx2-2 is dependent on imp $\beta 1$ or imp 13, but not imp 4 when incubated at $30^{\circ} \mathrm{C}(\mathrm{b}, \mathrm{C}$, d). GST-Nkx2-2-EGFP was not detected in the nuclei when incubated at $4^{\circ} \mathrm{C}(e, f, g)$. 
B

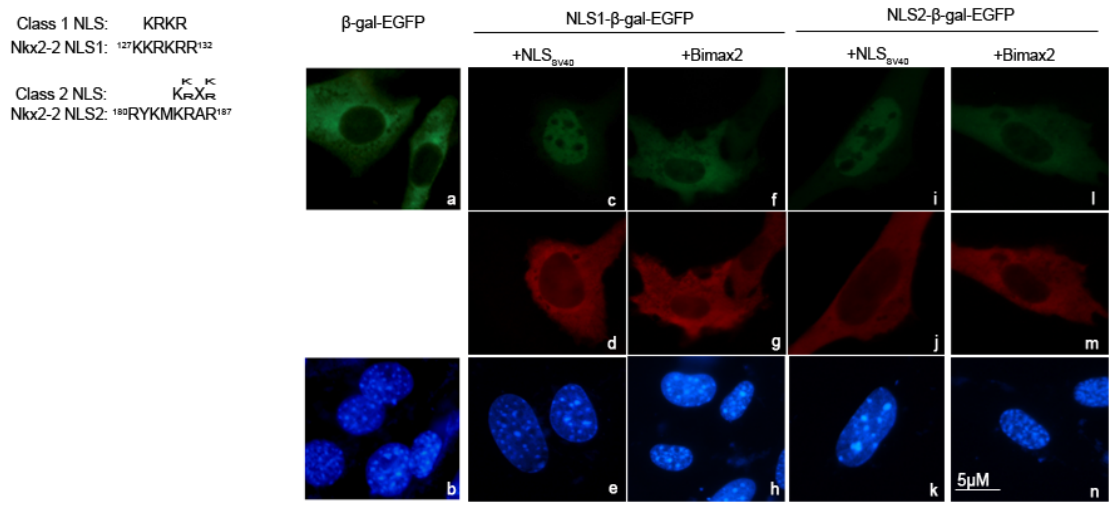


A

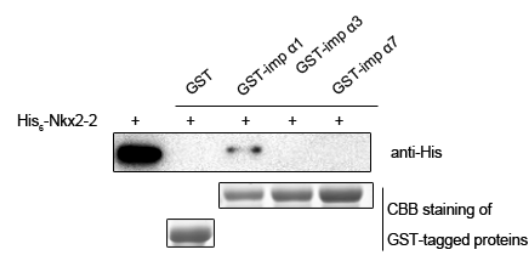

B

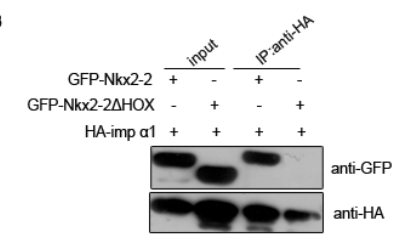

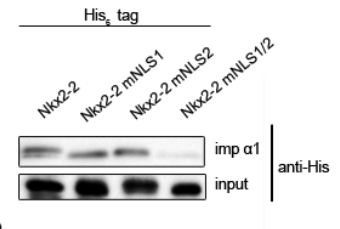
\begin{tabular}{cccc}
\multicolumn{4}{c}{ Nuclear import of GST-Nkx2-2-EGFP with Ran,Energy and: } \\
\hline Buffer & imp a $1 / \beta 1$ & imp a $3 / \beta 1$ & imp a $7 / \beta 1$
\end{tabular} 

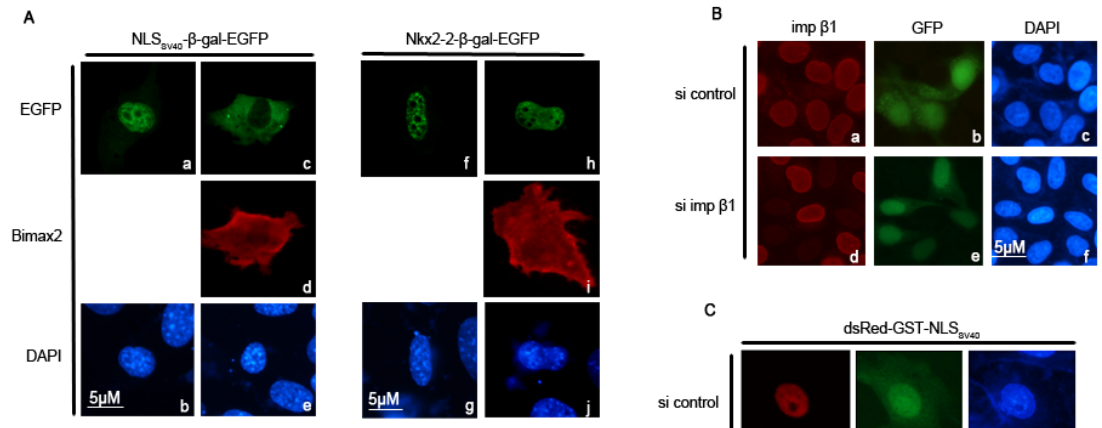

$\operatorname{simp\beta 1~}^{-}{ }^{+}{ }_{\text {imp } \beta 1}$

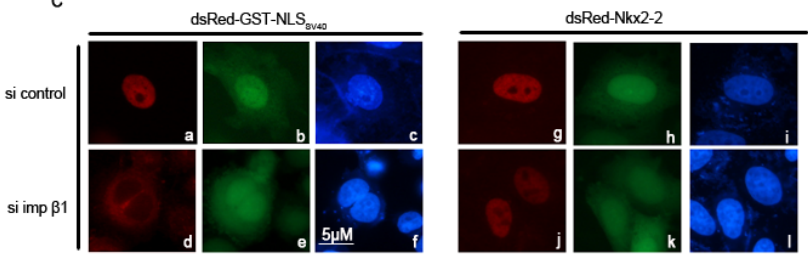



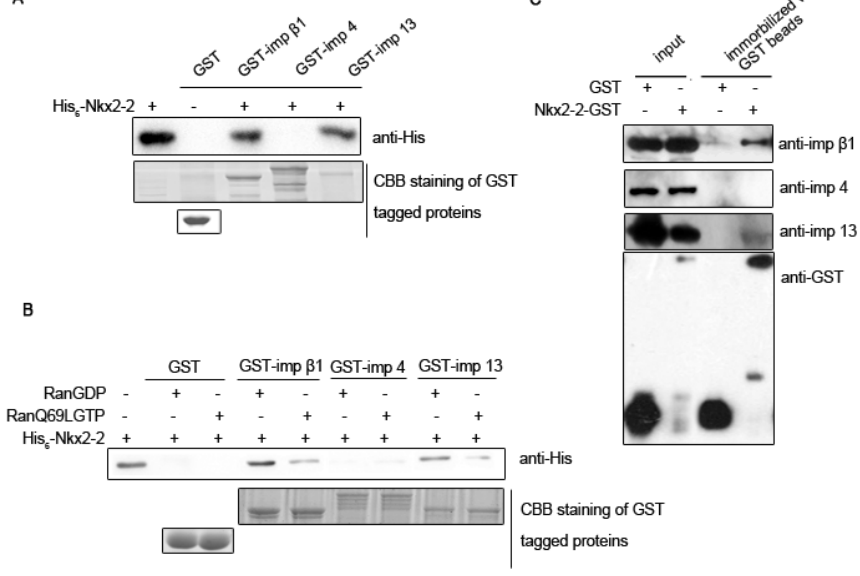

Nkx2-2-GST

- - $-a n t i-i m p \beta 1$

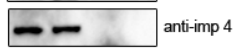

\begin{tabular}{cc} 
anti-imp 4 \\
\hline- & anti-imp 13
\end{tabular} c

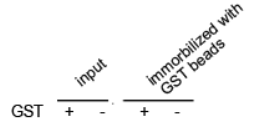

$=0$

EST
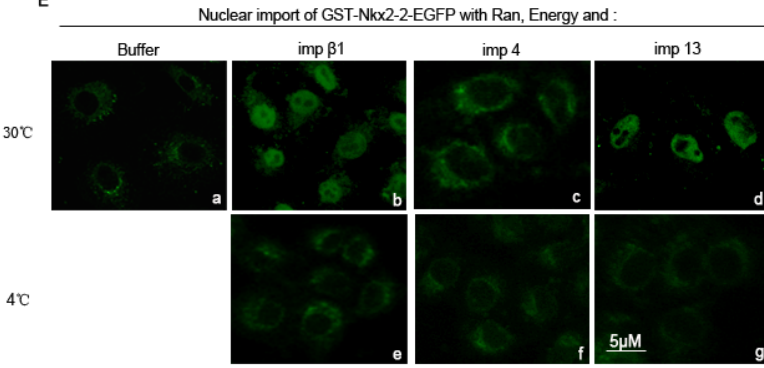